\title{
$J$ PREDICTIONS FOR DEFECTIVE PIPE ELBOWS VIA THE REFERENCE STRESS METHOD
}

\author{
Yuebao Lei ${ }^{1}$ \\ ${ }^{1}$ Structural Integrity Branch, EDF Energy, Barnett Way, Barnwood, Gloucestershire, GL4 3RS, UK \\ (yuebao.lei@edf-energy.com)
}

\begin{abstract}
Assessment of elbows with detected or postulated defects is required to support structural integrity related safety cases. In R6, the $J$-based failure assessment diagram (FAD) method is used in the fracture assessment, which is underpinned by the reference stress $J$ scheme. Therefore, an assessment using the R6 FAD method is equivalent to a $J$ prediction using the reference stress method. In this paper, the effect of various limit load solutions of the defective elbows on the $J$ predictions is investigated using the available three dimensional elastic-plastic finite element (FE) $J$ results in order to create guidance for users to follow when performing structural integrity assessment of defective elbows using the R6 procedure. The results show that using the global limit load may lead to reasonably conservative $J$ predictions. However, the availability of global limit load solutions is very limited. The results also show that using a local limit load can lead to conservative $J$ predictions for most of the cases. However, the estimated local limit load for some cases could be higher than the corresponding global limit load and result in non-conservative $J$ predictions. Future work may focus on the parameter optimisation of the local limit load model for complex structures.
\end{abstract}

\section{INTRODUCTION}

Elbows are widely used in nuclear power plant pipelines, both in nuclear and conventional islands. Assessment of elbows with detected or postulated defects is required to support structural integrity related safety cases and plant life extension arguments. In R6 (2019), the $J$-based failure assessment diagram (FAD) method is used in the fracture assessment, which is underpinned by the reference stress $J$ scheme (Ainsworth (1984)). Therefore, an assessment using the R6 FAD method is equivalent to a $J$ prediction using the reference stress method. The stress intensity factor (SIF) and the limit load of the defective structure are two most important inputs in the reference stress $J$ estimation and the R6 assessment. For defective pipe elbows, a simplified SIF estimation method has been recommended by Lei and Budden (2017). In this paper, the effect of various limit load solutions of the defective elbows on the $J$ predictions is investigated using the available three dimensional (3D) elastic-plastic finite element (FE) $J$ results in order to create guidance for users to follow when performing structural integrity assessment using the R6 procedure.

\section{ELBOW GEOMETRY AND LOAD DEFINITIONS}

For consistency, solutions from different sources are converted according to a uniform notation system used in this paper. The dimensions of a pipe elbow can be described by its elbow radius, $R_{\mathrm{c}}$, elbow angle, $\psi_{\mathrm{c}}$, pipe mean radius, $r_{\mathrm{m}}$, and pipe wall-thickness, $t$ (see Fig. 1 ). The elbow factor, $\lambda_{\mathrm{b}}$, is defined as

$$
\lambda_{\mathrm{b}}=\left(R_{\mathrm{c}} / r_{\mathrm{m}}\right) /\left(r_{\mathrm{m}} / t\right)
$$

The elbow factor, $\lambda_{\mathrm{b}}$, ratios $R_{\mathrm{d}} / r_{\mathrm{m}}$ and $r_{\mathrm{m}} / t$ and elbow angle, $\psi_{\mathrm{c}}$, are four important parameters to define a pipe elbow. When required, the inner and outer radii ( $r_{\mathrm{i}}$ and $r_{\mathrm{o}}$, respectively) may be obtained from $r_{\mathrm{m}}$ and $t$ as $r_{\mathrm{i}}=r_{\mathrm{m}}-t / 2$ and $r_{\mathrm{o}}=r_{\mathrm{m}}+t / 2$. The load types considered in this paper are internal pressure, $p$, in-plane bending moment, $M_{2}^{0}$, out-of-plane bending moment, $M_{3}^{0}$, and torsion moment, $M_{1}^{0}$, and Fig. 1 shows the 
positive directions of the moments. The elbows considered are all with long attached straight pipes at both ends. The moment loads are assumed to be transferred to the elbow body through the attached straight pipes. Two angular parameters, $\psi\left(0 \leq \psi \leq \psi_{\mathrm{c}}\right)$ and $\varphi(\pi \geq \varphi>-\pi)$, where $\psi$ is measured from the elbow end where moment loads are applied (transferred from the attached straight pipe, see Fig.1) and $\varphi$ is defined in the $\psi$-section plane and measured from the local coordinate-2, are used to define the defect location in the elbow. Any circumferential defects are located in the section defined by $\psi$ and the deepest point location is defined by $\varphi$. An axial defect location is also defined by $\varphi$ and $\psi$, where $\psi$ defines the

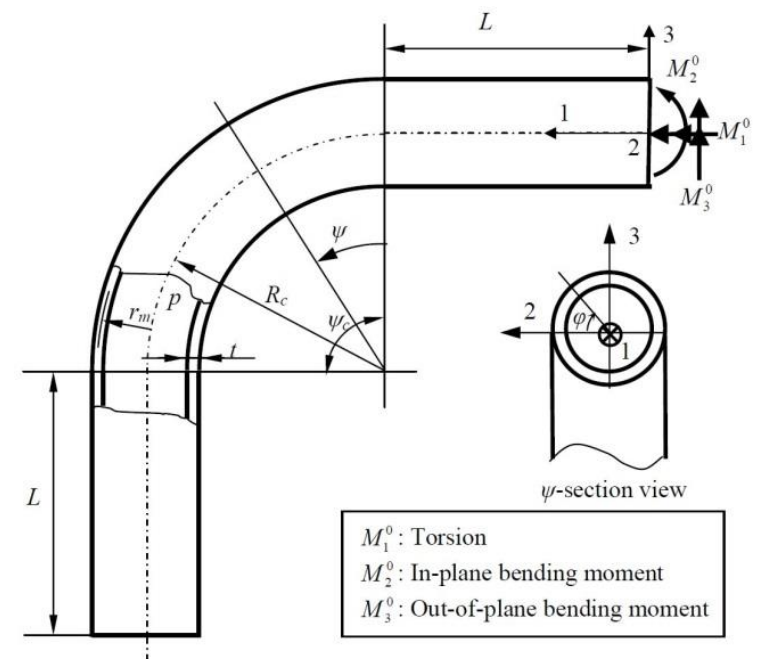

Fig. 1 Definition of elbow geometry parameters and loads

middle of the defect and $\varphi$ defines the defect location along the elbow pipe circumference. For an axial crack located at the intersection between the elbow and straight pipe $\left(\psi=0\right.$ or $\left.\psi=\psi_{c}\right)$, half of the crack is located inside the elbow and the other half is inside the straight pipe. For this case, the crack is not geometrically symmetric and the SIF and $J$ values at the two surface points of the crack may be different.

The defect types considered in this paper are semi-elliptical surface cracks. The depth of a semielliptical surface crack is indicted by $a$ and the half-length is represented by $c$. In this paper, defect types are represented by the following defect codes ( Kayser (2016)):

CDSI Circumferential internal semi-elliptical crack

CDSE Circumferential external semi-elliptical crack

LDSI Axial internal semi-elliptical crack

LDSE Axial external semi-elliptical crack

\section{REVIEW OF AVAILABLE LIMIT LOAD SOLUTIONS FOR DEFECTIVE PIPE ELBOWS}

The available limit load solutions for defective elbows have been reviewed and validated using the FE limit analyses by Lei (2011). Alternatively, Lei (2018) has made effort to use local limit load which is independent of loading types and can, therefore, be used for any load combinations. All these available solutions are considered in this investigation.

\section{The Available Global Limit Load Solutions}

Lei (2011) reviewed the limit load solutions for defective elbows and found that almost all the available global limit load solutions were based on FE limit load analyses. Due to the complexity of elbow geometry and various load types (pressure, in-plane/out-of-plane bending moment and torsion), the available solutions are very limited, especially for cases with load type combinations, and cannot satisfy the requirement of industrial structural integrity assessments. To fill the gap, an equivalent straight pipe method was proposed (Lei (2011)), based on the geometry and loading similarity between an elbow and 
the corresponding straight pipe, and validated using available FE limit load results for various elbow/crack geometry parameters and load types. The validation (Lei (2011)) showed that this method provided conservative global limit load solutions.

The equivalent straight pipe method can be described by

$$
\left(\frac{P_{L}}{P_{L 0}}\right)_{\text {elbow }}=\left(\frac{P_{L}}{P_{L 0}}\right)_{\text {straight pipe }}
$$

where $P_{L}$ and $P_{L 0}$ represents the limit load in general for a defective and defect-free component, respectively, of the same geometry under the same loading type or load combinations. The assumption of this method is that the limit load ratio between a defective and defect-free elbow may be approximately obtained from the limit load ratio between a defective and defect-free straight pipe with the same geometry and crack parameters under the same loading conditions at the defect section in the elbow. This means that the global limit load for a defective elbow may be evaluated using the available straight pipe limit load solutions. However, due to the availabilities of the limit load solutions for defective straight pipes, this method can only be used for elbows with circumferential internal/external surface cracks under internal pressure, $p$, in-plane bending, $M_{2}^{0}$, and combined internal pressure and in-plane bending $\left(p+M_{2}^{0}\right)$. For elbows with axial internal/external surface cracks, it can only be used for internal pressure.

For elbows with circumferential surface cracks under pressure, Eqn. (2) can be expressed as

$$
\frac{p_{L}}{p_{L 0}}=\frac{p_{L}^{S}}{p_{L 0}^{s}}
$$

where $p_{L}$ is the limit pressure for the defective elbow, $p_{L}^{S} / p_{L 0}^{S}$ is the limit pressure ratio between the defective and defect-free straight pipe for the same pipe and crack geometries under same type of loading, which can be evaluated using the R6 (2019) Section IV.1 solution, and $p_{L 0}$ is the limit load of the defectfree elbow, which can be expressed as (Lei (2011))

$$
\frac{p_{L 0}}{p_{0}}=c_{1}\left(\frac{R_{c}}{r_{m}}\right)^{n_{1}} \quad \text { for } 1.5 \leq \frac{R_{c}}{r_{m}}<6, \quad \frac{p_{L 0}}{p_{0}}=\frac{1-r_{m} / R_{c}}{1-r_{m} /\left(2 R_{c}\right)} \text { for } \frac{R_{c}}{r_{m}} \geq 6
$$

where $p_{0}=(2 / \sqrt{3})\left(t / r_{m}\right) \sigma_{y}, \sigma_{y}$ is the yield stress of material and parameters $c_{1}$ and $n_{1}$ can be evaluated using the following equation.

$$
\left\{\begin{array}{rr}
c_{1}=0.8447-0.0099\left(r_{m} / t\right) \text { and } n_{1}=0.0373-0.0075\left(r_{m} / t\right) & \text { for } 2 \leq r_{m} / t \leq 15 \\
c_{1}=0.6962 \text { and } n_{1}=0.1498 \quad \text { for } 15<r_{m} / t \leq 30
\end{array}\right.
$$

For elbows with circumferential surface cracks under in-plane bending moment, Eqn. (2) can be expressed as

$$
\frac{M_{L}}{M_{L 0}}=\frac{M_{L}^{S}}{M_{L 0}^{S}}
$$

where $M_{L}$ is the limit moment for the defective elbow, $M_{L}^{S} / M_{L 0}^{S}$ is the limit moment ratio between the defective and defect-free straight pipe for the same pipe and crack geometries under same type of loading, which can be evaluated using the R6 (2019) Section IV.1 solution, and $M_{L 0}$ is the limit moment of the defect-free elbow, which can be evaluated, for material with $0.001 \leq \sigma_{y} / E \leq 0.0015$ (where $E$ is Young's Modulus), from the following equations (Lei (2011)),

$$
\frac{M_{L 0}}{M_{0}}=\left(1+\frac{0.22}{\lambda_{b}^{1.028+0.12\left(R_{c} / r_{m}\right)}}\right)^{-1} \text { for } \lambda_{b} \leq 1 \text { and } \frac{M_{L 0}}{M_{0}}=\left(1+\frac{0.22}{\lambda_{b}^{1.313}}\right)^{-1} \text { for } \lambda_{b}>1
$$

for closing bending and 


$$
\left\{\begin{array}{l}
\frac{M_{L 0}}{M_{0}}=0.8908+0.2052 \ln \left(\lambda_{b}\right) \quad \text { for } 0.1 \leq \lambda_{b}<1 \\
\frac{M_{L 0}}{M_{0}}=\left(1+0.45 \exp \left(-1.3 \lambda_{b}\right)\right)^{-1} \text { for } 1 \leq \lambda_{b} \leq 1.5
\end{array}\right.
$$

for opening bending, where $M_{0}=4 r_{m}^{2} t \sigma_{y}$.

For elbows with circumferential surface cracks under combined pressure and in-plane bending, it is assumed that the $M_{L} / M_{L 0} \sim p_{L} / p_{L 0}$ relationship for elbows, $M_{L} / M_{L 0}=f\left(p_{L} / p_{L 0}\right)$, is the same as the $M_{L}^{S} / M_{L 0}^{S} \sim p_{L}^{S} / p_{L 0}^{S}$ relationship for straight pipes, $M_{L}^{S} / M_{L 0}^{S}=f\left(p_{L}^{S} / p_{L 0}^{S}\right)$, which can be obtained from the R6 (2019) Section IV.1. For proportional loading, the limit in-plane bending moment and the limit pressure can be obtained by solving the $M_{L} / M_{L 0}=f\left(p_{L} / p_{L 0}\right)$ equation.

For axial internal/external surface cracks under pressure, the limit pressure for a defective elbow, $p_{L}$, can be evaluated from the following equation,

$$
\frac{p_{L}}{p_{L 0}}=\left(\frac{p_{L}^{S}}{p_{L 0}^{S}}\right)\left(\frac{F_{S}}{p_{L 0} / p_{0}}\right) \frac{1+\left(r_{m} / R_{c}\right) \sin \varphi}{1+\left(r_{m} /\left(2 R_{c}\right)\right) \sin \varphi} \leq 1, \text { otherwise } \frac{p_{L}}{p_{L 0}}=1
$$

where $F_{S}$ is defined by the following two equations

$$
\begin{aligned}
& \begin{cases}F_{S}=1 & \text { for } 0 \leq \varphi \leq \pi \\
F_{S}=1-\frac{2}{\pi}\left(F_{1}-1\right) \varphi & \text { for } 0>\varphi \geq-\frac{\pi}{2} \\
F_{S}=1+\frac{2}{\pi}\left(F_{1}-1\right)(\pi+\varphi) & \text { for }-\frac{\pi}{2}>\varphi \geq-\pi\end{cases} \\
& F_{1}=\left(p_{L 0} / p_{0}\right)\left[1-\left(r_{m} /\left(2 R_{c}\right)\right)\right] /\left[1-\left(r_{m} / R_{c}\right)\right]
\end{aligned}
$$

The straight pipe solution, $p_{L}^{S} / p_{L 0}^{S}$, in Eqn. (9) can be obtained from R6 (2019) Section IV.1.

\section{The Local Limit Load Solution}

A new local limit load model for shell/plate type components (plane stress conditions along the thickness) with surface cracks was developed by Lei (2018). The model (see Fig. 2) is a plate of width $2 D$ which contains a rectangular surface crack of depth $a$ and length $2 c$ circumscribing the real surface defect and has the same thickness, $t$, as the component at the crack location. The plate is assumed to be sufficiently long (this could be different from the real structure to be simulated). The half width of the plate, which should be less than the half width of the component, $W$, is defined as

$$
D=(2+k(a / t)) c<W
$$

where $k$ is a constant defined for different geometries ( $k=1.5$ for plates and 1 for cylinders and elbows). For elbows, $W$ can be the half length of the elbow measured from the middle of the crack for axial cracks and the half circumference of the elbow pipe for circumferential cracks.

The model is remotely loaded by the primary stresses of the component at the crack location obtained from elastic uncracked-body stress analysis. The stresses obtained from the elastic stress analysis should be expressed as the membrane stress, $\sigma_{\mathrm{m}}$, and through-thickness bending stress, $\sigma_{\mathrm{b}}$, normal to the crack plane, the membrane stress, $\sigma_{2 \mathrm{~m}}$, parallel to the crack plane and the possible average shear stress in the crack plane, $\tau_{\mathrm{m}}$. The through-thickness bending stress, $\sigma_{\mathrm{b}}$, is positive if bending stress tends to open the surface crack and may be ignored when it is judged to be a non-primary stress. The reference stress of the model can be expressed as

$$
\sigma_{\mathrm{ref}}=\sqrt{\left(3 \tau_{\mathrm{m}}^{2}+\left(\sigma_{\mathrm{ref}}\right)_{\tau_{m}=0}^{2}\right)}
$$

where 


$$
\left(\sigma_{\text {ref }}\right)_{\tau_{\mathrm{m}}=0}= \begin{cases}\frac{\sigma_{\mathrm{m}}}{n_{\mathrm{L}}\left(\lambda, \lambda_{1}\right)} & \text { for } \sigma_{\mathrm{m}} \neq 0 \\ \frac{\sigma_{\mathrm{b}}}{m_{\mathrm{L}}\left(\lambda_{2}\right)} & \text { for } \sigma_{\mathrm{m}}=0\end{cases}
$$

where $n_{\mathrm{L}}$ and $m_{\mathrm{L}}$ are normalised limit membrane stress and limit bending stress under combined loading, respectively, which can be calculated using the solutions given in Lei and Budden (2015) with

$$
\lambda=\sigma_{b} /\left(6 \sigma_{m}\right), \lambda_{1}=\sigma_{2 m} / \sigma_{m}, \lambda_{2}=\sigma_{2 m} / \sigma_{b}
$$

The reference stress of Eqn. (13) is then used to define $L_{r}$ (see Eqn. (17) below) in the $J$ prediction or defect assessment.

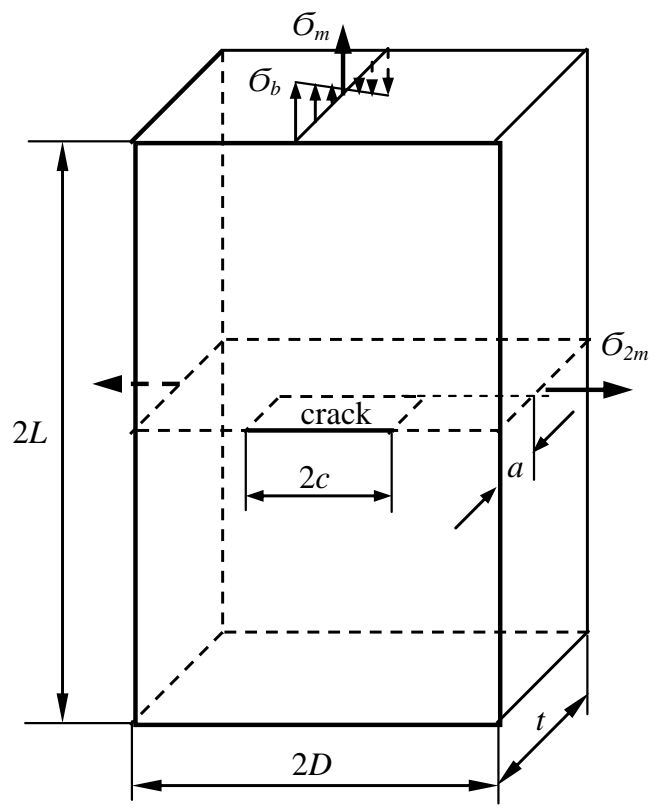

Fig. 2 The local limit load model

\section{VALIDATION OF LIMIT LOAD SOLUTIONS USING THE FE $\boldsymbol{J}$ RESULTS}

The global limit load solutions and the developed local limit load solution may be validated by comparing the $J$ values predicted using the obtained reference stress via the reference stress $J$ prediction method and the elastic-plastic FE results. A reference stress solution is conservative if the FE $J$ values are overestimated when it is used in the reference stress $J$ scheme. In this paper, well-documented $J$ solutions from the published literature are collected, reviewed and used to validate the global and local limit load solutions in order to develop guidance for users to follow in defective elbow assessment.

\section{The Reference Stress J Estimation Method}

The reference stress $J$ estimation scheme predicts the total $J$ from the elastic $J, J_{\mathrm{e}}$, using the reference stress, $\sigma_{\text {ref }}$, and corresponding reference strain, $\varepsilon_{\text {ref }}$, via the following relationship based on the R6 Option 2 failure assessment curve (FAC) (R6 (2019))

$$
\frac{J}{J_{\mathrm{e}}}=\frac{E \varepsilon_{\mathrm{ref}}}{\sigma_{\mathrm{ref}}}+\frac{L_{r}^{2}}{2} \frac{\sigma_{\mathrm{ref}}}{E \varepsilon_{\mathrm{ref}}}
$$

where the reference stress and strain follow the uniaxial stress-strain relationship of the material and $L_{\mathrm{r}}$ may be defined by the limit load or the reference stress via

$$
L_{\mathrm{r}}=\sigma_{\mathrm{ref}} / \sigma_{\mathrm{y}}
$$


where $\sigma_{\mathrm{y}}$ is the yield stress or $0.2 \%$ plastic strain offset stress of the material.

\section{The Available FE J Results and Validation Method}

Elastic and elastic-plastic fracture analyses for elbows with axial/circumferential internal/external semielliptical cracks under internal pressure and bending moment(s) and some load combinations (Kayser (2016)) were performed and the elastic and elastic-plastic $J$ values are available and can be used to validate the elbow limit load solutions via the reference stress $J$ scheme. The geometry and loading information for the cases analysed is summarised in Table 1. Real material properties were used in the FE analyses with $E=175 \mathrm{GPa}, v=0.3, \sigma_{0.2}=184.8 \mathrm{MPa}$ and the strain hardening exponent $n=6$.

For each case listed in Table 1, $J$ values are predicted using the reference stress method via Eqn. (16) using the material true stress-true strain data. The results are then compared with the corresponding available FE values at the estimated $L_{\mathrm{r}}$ for a selected limit load solution. A good limit load solution should lead to a conservative but reasonably accurate prediction compared with the FE $J$ results.

Table 1: Cases with elastic-plastic $J$ results [Kayser (2016)]

\begin{tabular}{|c|c|c|c|c|c|c|c|c|}
\hline Cases & CC-1 & CC-2 & CC-3 & CC-4 & AC-1 & AC-2 & AC-3 & AC-4 \\
\hline Code & CDSI & CDSI & CDSI & CDSI & LDSI & LDSE & LDSI & LDSI \\
\hline$R_{c} / r_{m}$ & 6 & 4 & 4 & 6 & 4 & 4 & 4 & 4 \\
\hline$r_{m} / t$ & 10 & 10 & 10 & 10 & 10 & 10 & 10 & 10 \\
\hline$\psi_{c}\left(^{\circ}\right)$ & 90 & 90 & 90 & 90 & 90 & 90 & 45 & 90 \\
\hline$\psi\left(^{\circ}\right)$ & 0 & 0 & 0 & 0 & 45 & 45 & 22.5 & 45 \\
\hline$\varphi\left(^{\circ}\right)$ & 90 & 90 & 171 & 177 & 90 & -90 & -90 & -90 \\
\hline$a / t$ & 0.25 & 0.25 & 0.25 & 0.25 & 0.25 & 0.25 & 0.25 & 0.25 \\
\hline$a / c$ & 0.33 & 0.33 & 0.33 & 0.33 & 0.33 & 0.33 & 0.33 & 0.33 \\
\hline Load(s) & $p$ & $M_{2}^{0}$ & $M_{3}^{0}$ & $M_{3}^{0}+M_{1}^{0}$ & $p$ & $M_{2}^{0}$ & $\mathrm{p}+M_{2}^{0}$ & $M_{2}^{0}+M_{3}^{0}$ \\
\hline $\begin{array}{c}\text { Global } \\
\text { limit } \\
\text { load? }\end{array}$ & Yes & Yes & $\begin{array}{c}\text { Yes } \\
\text { (approximated) }\end{array}$ & No & Yes & No & No & No \\
\hline
\end{tabular}

\section{COMPARISON OF $\boldsymbol{J}$ VALUES BETWEEN FE AND PREDICTIONS WITH VARIOUS LIMIT LOADS}

For each case with available FE $J$ results, elastic uncracked-body stress analysis is carried out using the elbow elastic nominal stress calculation method proposed by Marie et al. (2007) to determine the required model loads for the local limit load model and the local limit load based reference stress, $\sigma_{\text {ref }}$, is evaluated from Eqn. (13). For global limit load, the reference stress is determined from Eqn. (18) below.

$$
\frac{\sigma_{\text {ref }}}{\sigma_{\mathrm{y}}}=\frac{\text { Primary load }}{\text { Global limit load }}
$$

The FE $J / J_{e}$ values are plotted against $\sigma_{r e f} / \sigma_{y}$ determined from local limit load solution (denoted by "FE, local limit load") and available global limit load solutions (denoted by "FE, global limit load") in Fig. 3 for circumferential surface cracks and Fig. 4 for axial surface cracks, where $J_{e}$ is obtained from SIF solutions determined by using the method recommended by Lei and Budden (2017). For all the cases shown in Figs. 3 and 4 the predicted $J / J_{e}$ results using Eqn. (16) based on the material true stress-true strain data are also plotted in the each figure for comparison, where the predicted results are indicated by "Predicted (Opt-2 FAC)" in the figures. 


\section{DISCUSSION}

\section{J Predictions Using The Global Limit Load Solutions}

There are only four out of eight cases in Figs. 3 and 4 with global limit load available, three cases for circumferential cracks and one case for axial cracks. From the figures, the predicted $J$ values are reasonably accurate and conservative for all the four cases, even for the cases with the FE based global limit load (see Figs. 3(a) and (b), denoted by "FE, global limit load (FE based)"), where the global FE based limit load in Fig. 3(a) is due to Hong et al. (2009) and that in Fig. 3(b) is due to Kim et al. (2007).

For the cases with circumferential cracks, Eqns. (3) and (6) have some degree of in-built conservatism compared with the FE plastic collapse load data (see Lei (2011)). This could contribute to the conservatism in $J$ prediction. Another reason could be due to the difference in crack location between the limit load solutions and the cases of this paper. Both Eqns. (3) and (6) and the FE based solutions (Hong et al. (2009) and Kim et al. (2007)) used in this paper are for a crack in the middle of the elbow ( $\psi$ $=\psi_{\mathrm{c}} / 2$ ). However, all the circumferential cracks in elbows considered in this paper are located at the intersection between the elbow and the attached straight pipe $(\psi=0)$. Note that the weakest point of an elbow is at the middle of the bend. Therefore, using the limit load solutions for cracks located at the middle of the elbow to the cases where crack is located at the intersection between the elbow and the attached straight pipe is a conservative consideration. However, if the FE global limit load based on the plastic collapse mechanism at the intersection between the elbow and the attached straight pipe is used,

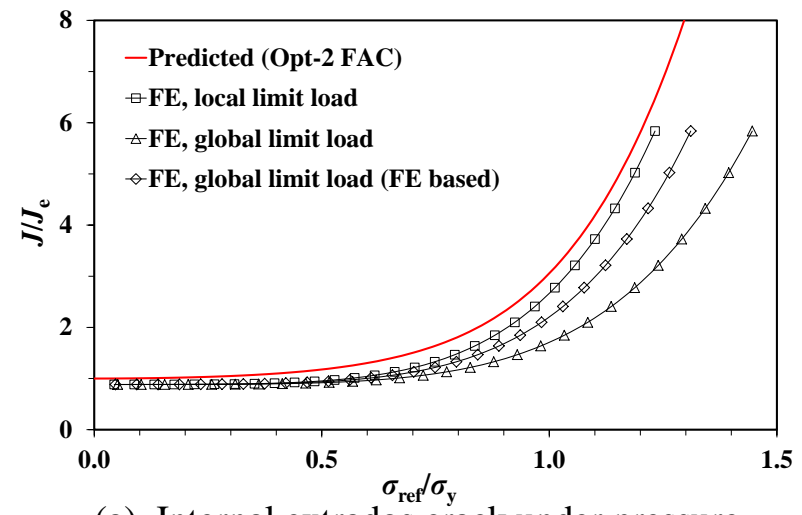

(a) Internal extrados crack under pressure

(Case CC-1)

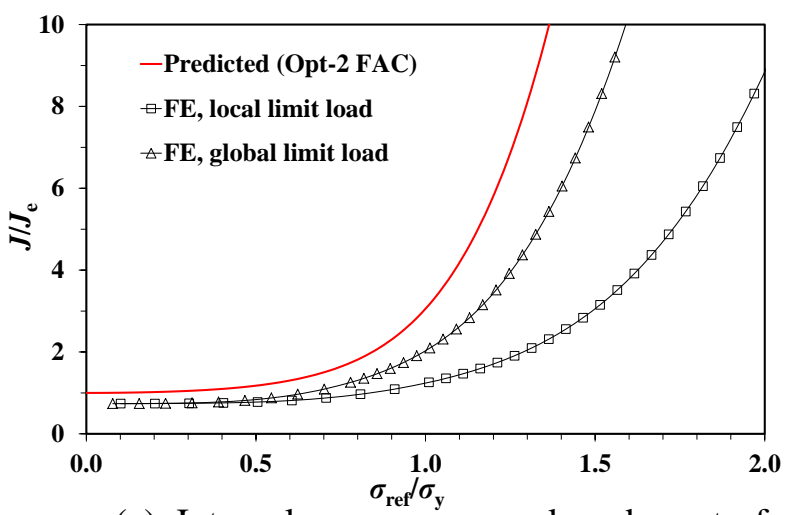

(c) Internal near crown crack under out-ofplane bending (Case CC-3)

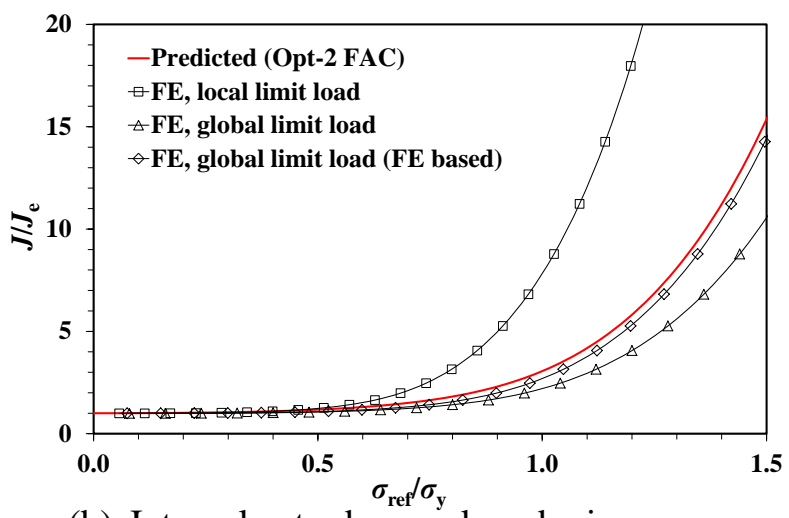

(b) Internal extrados crack under in-pane bending (Case CC-2)

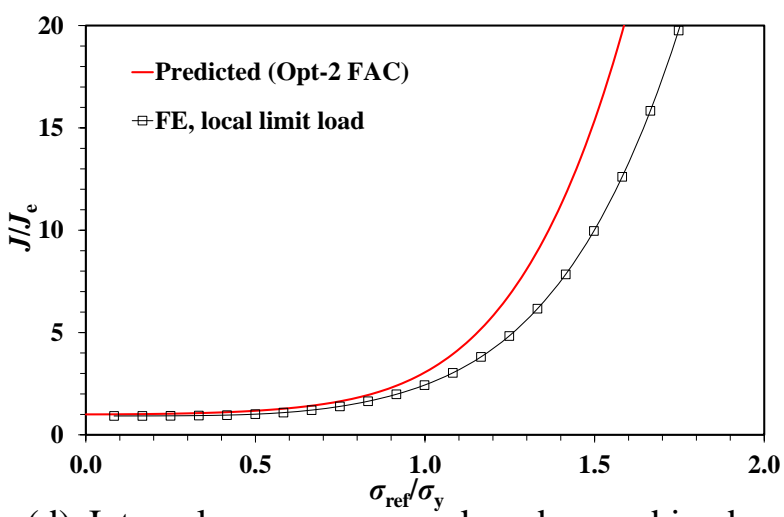

(d) Internal near crown crack under combined out-of-plane bending and torsion (Case CC-4)

Fig. 3 Comparison of $J$ values between FE results and the reference stress method predictions using various limit loads for circumferential semi-elliptical surface cracks in elbows 


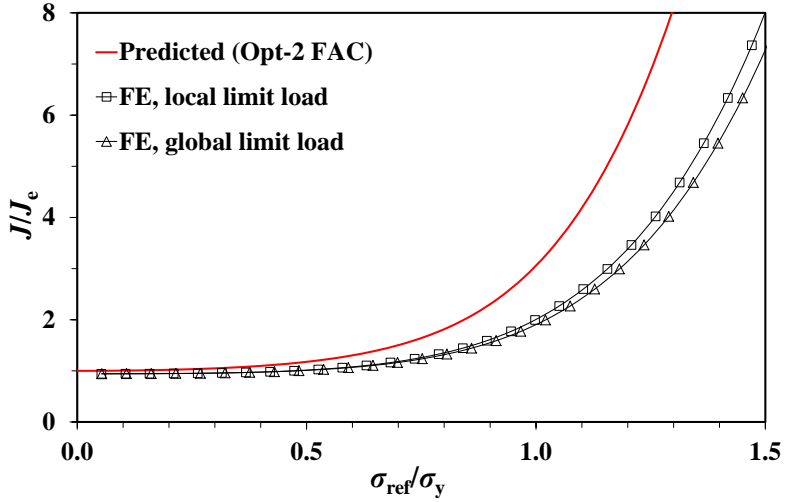

(a) Internal extrados crack under pressure (Case AC-1)

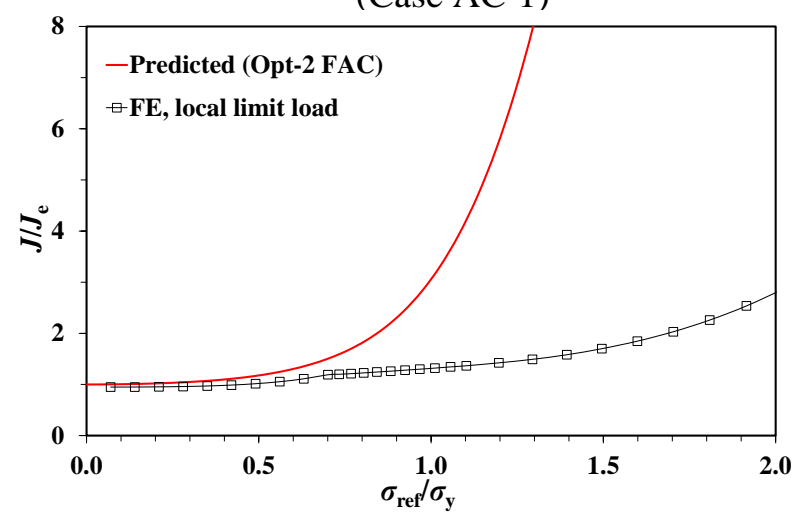

(c) Internal intrados crack under combined pressure and in-plane bending (Case AC-3)

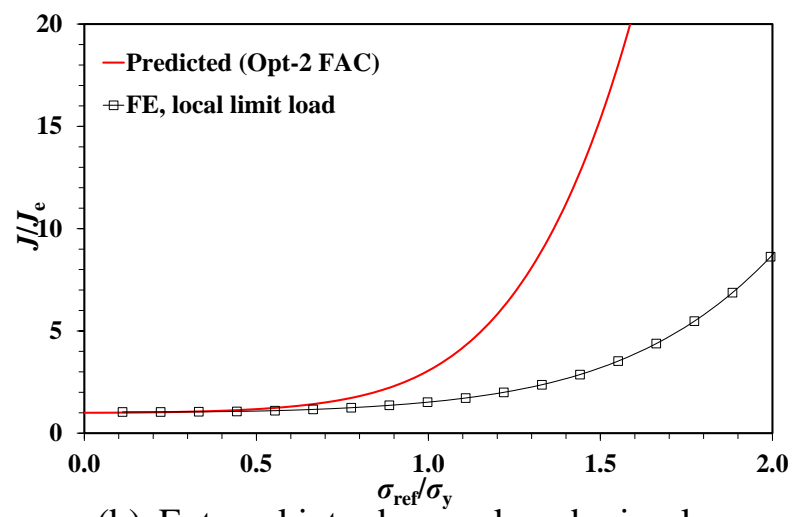

(b) External intrados crack under in-plane bending (Case AC-2)

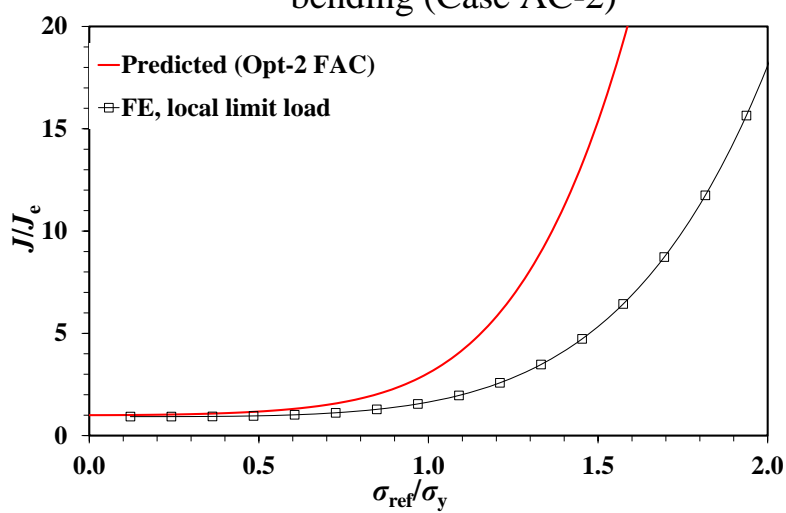

(d) Internal extrados crack under combined in-plane and out-of-plane bending (Case AC-4)

Fig. 4 Comparison of $J$ values between FE results and the reference stress method predictions using various limit loads for axial semi-elliptical surface cracks in elbows

the predictions may be more accurate but the conservatism cannot be guaranteed.

For the case with an axial surface crack (Fig. 4(a)), Eqn. (6) also bears some degree of conservatism compared with the FE plastic collapse data (see Lei (2011)) and, therefore, can lead to conservative $J$ predictions when used in the reference stress method.

In conclusion, the global limit load solutions for circumferential surface cracks in elbows under pressure (Eqn. (3)) or in-plane bending (Eqn. (6)) and for axial surface cracks in elbows under pressure (Eqn. (9)) recommended by Lei (2011) can lead to reasonably accurate and conservative $J$ predictions via the reference stress method.

\section{J Predictions Using The Local Limit Load Solution}

The local limit load method applies all the cases listed in Table 1 because the local limit load is determined from the uncracked body stresses at the crack location regardless of the remote load types and load combinations.

For cases with circumferential semi-elliptical surface cracks, from Fig. 3, reasonably conservative $J$ predictions have been obtained using the reference stress estimation based on the local limit loads for three cases out of four. For internal pressure, the predictions are very accurate but for out-of-plane bending moment, the predictions are more conservative. The predictions for the case of in-plane bending moment are non-conservative (see Fig. 3(b)). From Fig. 3(b), for a given load level, the $\sigma_{\text {ref }} / \sigma_{\mathrm{y}}$ estimated 
using the local limit load is lower than these using the global limit loads, i.e., the local limit load is significantly higher than the global limit load defined by Eqn. (6) and the FE based limit load solution. This could be due to the very strong through-thickness bending stress on the two surfaces where the membrane stress parallel to the crack plane, $\sigma_{2 \mathrm{~m}}$, applies (see Fig. 2). Currently, there is no such limit load solution to include the effect of this bending stress for the local limit load model and the average bending stress distribution along the crack ligament is treated as an equivalent membrane stress which is then added to $\sigma_{2 \mathrm{~m}}$. It is clear that this treatment is not good enough to describe the effect of the bending stress parallel to the crack plane on the limit load of the local limit load model.

For the cases with axial semi-elliptical surface cracks, from Fig. 4, conservative $J$ predictions have been obtained for all the four cases. For internal pressure (Fig. 4(a)), the predictions are reasonably accurate and conservative. For this case, the through-thickness bending stress is small and bi-axial membrane stresses dominate. Figure 4(d) shows the results for combined in-plane and out-of-plane loading, which causes in-plane, out-of-plane and torsion at the cross section of the crack location. For this very complex loading case the $J$ predictions with reasonable conservatism are acceptable. However, for in-plane bending (Fig. 4 (b)) and combined pressure and in-plane bending (Fig. 4(c)), the predictions are very conservative. For these cases, through-thickness bending stresses dominate and the opening crack membrane stress, $\sigma_{\mathrm{m}}$, is very small. Note that the through-thickness bending stresses have secondary stress nature and may relax due to existence of crack and local plastic deformation. Currently, the through-thickness bending stresses are treated as primary and the real limit load is clearly underestimated for these cases.

In conclusion, the local limit load method based on the local limit load model due to Lei (2018), can lead to reasonably conservative $J$ predictions for most of the cases considered but the estimated local limit load could be higher than the corresponding global limit load for some cases and lead to nonconservative $J$ predictions. Further investigation is required to improve the local limit load model. The future work may be focused on the model parameter optimisation, such as the definitions of half model width $D$ and $k$, and the treatment of through-thickness bending stresses in different way.

\section{CONCLUSIONS}

The effect of limit loads on the $J$ prediction of defective elbows via the reference stress method has been investigated by compare the reference stress predictions with 3-D finite element (FE) $J$ calculations for axial/circumferential internal/external semi-elliptical cracks in smooth pipe elbows. Available global and local limit load solutions have been considered. The results show that the global limit load solutions give the best predictions in general and the recommended limit load solutions shown in this paper with in-built conservatism can lead to reasonably conservative predictions. The FE based global limit load solutions may give more accurate $J$ predictions but the results could be non-conservative. The local limit load, based on the local limit load model due to Lei (2018), can lead to reasonably conservative $J$ predictions for most of the cases considered but the estimated local limit load could be higher than the corresponding global limit load for some cases and lead to non-conservative $J$ predictions. Further investigation on the parameter optimisation and stress classification of the local limit load model for cases with strong through-thickness bending stresses will be carried out in future.

\section{ACKNOWLEDGMENTS}

The author wishes to acknowledge Dr C. J. Aird of EDF Energy for his valuable comments on this paper. This paper is published by permission of EDF Energy Nuclear Generation Ltd.

\section{REFERENCES}

Ainsworth, R. A. (1984). "The assessment of defects in structures of strain hardening material", Engineering Fracture Mechanics 19, 633-642.

Hong, S.-P., Kim, J.-H., Kim, Y.-J. (2009). "Limit pressures of $90^{\circ}$ elbows with circumferential surface cracks", Engineering Fracture Mechanics. 76, 2202-2216. 
Kayser, Y. (2016). "BENCH KJ: overall synthesis of OECD benchmark activities", CEA Report DEN/DANS/DM2S/SEMT/LISN/NT/15-011/A.

Kim, Y.-J., Kim, Y.-I., Song, T.-K. (2007). "Finite element plastic loads for circumferential cracked pipe bends under in-plane bending", Engineering Fracture Mechanics 74, 643-668.

Lei, Y. (2011). "Review of limit load solutions for defective pipe bends", EDF Energy Report E/REP/BBGB/0060/GEN/09 Revision 0. EDF Energy Nuclear Generation Ltd., Gloucestershire, $\mathrm{UK}$.

Lei, Y. (2018). "A local limit load model for J prediction via the reference stress method", Procedia Structural Integrity, 13 571-577.

Lei, Y. and Budden, P. J. (2015). "Global limit load solutions for plates with surface cracks under combined biaxial forces and cross-thickness bending", International Journal of Pressure Vessels and Piping 132-133, 10-26.

Lei, Y. and Budden, P. J. (2017). "Stress intensity factor estimation for defective pipe elbows", SMiRT 24, Busan, Korea - August 20-25, 2017.

Marie, S., Chapuliot, S., Kayser, Y., Lacire, M. H., Drubay, B., Barthelet, B., Le Delliou, P., Rougier, V., Naudin, C., Gilles, P. and Triay, M. (2007). "French RSE-M and RCC-MR code appendices for flaw analysis: Presentation of the fracture parameters calculation-Part IV: Cracked elbows", International Journal of Pressure Vessels and Piping, 84, p.659-686, 2007.

R6 (2019). "Assessment of the Integrity of Structures Containing Defects", Revision 4, Amendment 12, EDF Energy Nuclear Generation Ltd., Gloucestershire, UK. 